\title{
Application of Artificial Immune System to Domestic Energy Management Problem
}

\author{
María Navarro-Cáceres*, Amin Shokri Gazafroudi*, Francisco Prieto-Castillo*†, Kumar G. Venyagamoorthy ${ }^{\ddagger}$ \\ and Juan Manuel Corchado* \\ *University of Salamanca \\ Espejo, s/n. Salamanca, 37003, Spain \\ ${ }^{\dagger}$ MediaLab, Massachusetts Institute of Technology \\ 20 Amherst St, Cambridge, Massachusetts, USA \\ ${ }^{\ddagger}$ Real-Time Power and Intelligent Systems Laboratory \\ Holcombe Department of Electrical and Computer Engineering, Clemson University \\ Clemson, SC 29634S, USA
}

\begin{abstract}
The connection of devices in a smart home should be done optimally, this helps save energy and money. Numerous optimization models have been applied, they are based on fuzzy logic, linear programming or bio-inspired algorithms. The aim of this work is to solve an energy management problem in a domestic environment by applying an artificial immune system. We carried out a thorough analysis of the different strategies that optimize a domestic environment system, in order to demonstrate the ability of an artificial immune system to find a successful optima that satisfies the problem constraints.
\end{abstract}

\section{INTRODUCTION}

Over the last decade, domestic buildings equipped with communication channels (commonly termed smart homes) have been involved in electrical grids as active players [1]. They constitute the building blocks -or prosumers (i.e. both consumers and producers)- in smart grids (SGs), and have an important role in the optimization of electrical energy scheduling [1], [2]. A Domestic Energy Management System (DEMS) is a crucial element in a smart home that improves the economy necessary through automation technologies.

There is a number of different strategies that optimize the scheduling of home power usage. Several approaches used different statistical models to improve DEMS problems. In particular, [3] models the controllable loads and the loads that depend on weather conditions using a Markovian approach. In [4], a demand response program that pursues classical methods has been applied automatically to the controllers used and the appliances controlled under uncertainty of outdoor temperature and electricity price. In [5], three methods of DEMS have been solved by applying an observable Markovian decision process which reduces the domestic energy costs in the time-varying electricity price market.

Due to the limitations of classical approaches, different paradigms have been developed to solve optimization problems. One of the most successful approaches is based on bioinspired algorithms. Bio-inspired algorithms imitate biological behavior to find solutions, otherwise too expensive to be obtained through classical computing in terms of time and resources. Among them, artificial neural networks, genetic algorithms and swarm intelligence are widely known [6]. In smart grids optimization, several works have been proposed. [7] addresses an energy service modeling method based on the Particle Swarm Optimization (PSO) algorithm. [8] proposes a multi-objective genetic approach to domestic load scheduling in an energy management system. [9] presents an Artificial Neural Network with a Genetic Algorithm (ANN-GA) smart appliance scheduling approach for optimized energy management in the domestic sector.

The application of one bio-inspired method such as the artificial immune system (AIS) has also brought good results in different contexts. There are some preliminary achievements in energy management, such as the energy dispatch problemsolving [10], or electrical reconfigurations [11]. In [12] an AIS is used to control thermal units in residential buildings and in [13] the authors optimize a wind-thermal generating system also with an AIS. However, AIS has never been applied to domestic environments to optimize profit and energy consumption.

Drawing on the positive results obtained with the AIS in similar problems, we present a new approach to optimize a domestic energy management problem using this algorithm. We demonstrate that AIS can be successfully applied to electric management problems in domestic environments. Among the different AIS variants, we selected Opt-aiNet [14], which has been used for function optimization successfully [14] in different contexts.

We present a preliminary electrical context in which the following different devices are found; a PV panel, a battery system, a space heater, a storage water heater, as well as mustrun services. They all are connected to each other in a smart home, forming a domestic electricity system. Our main goal is to optimize the schedule for the next 24 hours to maximize the electrical profit between the energy sold and the energy that we have to buy in order to maintain all these devices running correctly.

To validate the application of an AIS, we propose two strategies representing two different electrical situations. In Strategy 1, the DEMS manages electrical energy with power 
grid without considering any strict inside constraint. In other words, maintaining the home's electrical load through electrical energy produced by the PV system is not considered as a concern in Strategy 1. Thus, this strategy only pursues to optimize its energy profit.

However, in Strategy 2, the main goal of the DEMS is to supply the electrical demand autonomously whenever possible. Therefore, the surplus of the PV power generation is stored in the battery. Then, the DEMS will sell power to the grid if the battery is charged completely. Likewise, the battery is discharged if the electrical demand is more than power generation of the PV. Additionally, if the battery cannot supply all the electrical load, the DEMS will buy the electricity from the power grid.

We have adapted the opt-aiNet algorithm to include complex constraints in the optimization problem and to work efficiently with a large number of variables. We developed three different case studies, comparing AIS with a classical genetic algorithm (GA), comparing both strategies, and analysing different situations when the battery is involved in the grid. All the results validate the AIS as a proper algorithm for DEMS optimization.

This paper is structured as follows. Section II describes the technical details of the electrical problem addressed herein. Section III presents an overview of the AIS structure and design. The selected AIS is configured and applied to the concrete electrical problem in Section IV. In Section V we summarise our results. Finally, the conclusions from our research are presented in Section VI.

\section{Domestic Energy Management Problem}

The considered electrical context represents a domestic electrical system, where some appliances are connected. The proposed domestic electrical system is shown in Figure 1.

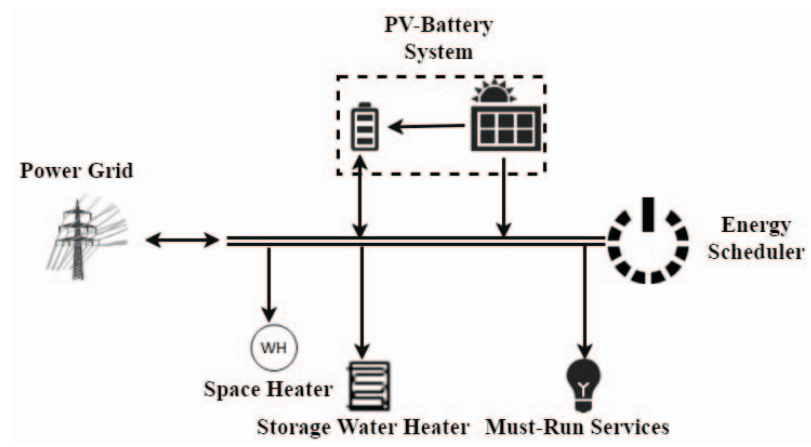

Fig. 1: Schematic image of domestic electrical system.

Our domestic grid considers appliances that can be classified into two parts: PV system, that contains the PV generator and the battery and Electrical Loads, which includes the space heater or air conditioner, the storage water heater, and the must-run services, representing loads that have to be supplied at any time. The appliances are connected to a power grid which can provide electrical load when the system requires so. The scheduler is responsible for balancing the profit of energy services, considering the PV, the power grid and the battery as providers of energy, and the rest of devices connected as consumers.

The objective of this problem is to maximize the profit of energy services provided in a domestic energy management system. Equation (1) expresses the term, $O F$, that is the objective function to optimize.

$$
\begin{aligned}
& O F=\sum_{t}\left(\lambda_{\text {sold }} P_{\text {sold }_{t}}-\lambda_{\text {bought }} P_{\text {bought }_{t}}\right. \\
& \left.-\sum_{j \in E L s} V O L L_{j} L_{j_{t}}^{s h e d}-V_{p v}^{s} S_{p v_{t}}\right)
\end{aligned}
$$

This function consists of four parts. First part, $\lambda_{\text {sold }} P_{\text {sold }}$, represents the revenue of selling energy produced by $\mathrm{PV}$ panels to the power grid. The total cost of electrical energy that is bought from the network, $\lambda_{\text {bought }} P_{\text {bought }}$, is represented in the second term. The value of electrical energy which is not served is stated in the third part, $\sum_{j \epsilon E L s} V O L L_{j} L_{j_{t}}^{s h e d}$, . Finally, the spillage costs of PV panels, $V_{p v}^{s} S_{p v_{t}}$, are represented in the last term of the equation.

The power balance equation is presented in (2). The power flow limitation through the distribution line is stated in (3).

$$
\begin{aligned}
& P_{\text {bought }_{t}}+P_{\text {pv }_{t}}+P_{b, \text { out }_{t}}=\sum_{j \in E L s}\left(L_{j_{t}}-L_{j_{t}}^{\text {shed }}\right)+P_{b, \text { in }_{t}} \\
& -f_{\text {max }} \leq P_{\text {bought }_{t}}-P_{\text {sold }_{t}} \leq f_{\text {max }}
\end{aligned}
$$

Additionally, the specific definitions for all domestic appliances are described in the following subsections.

\section{A. PV System}

The power output of PV system, $P_{p v_{t}}$, is obtained through (4).

$$
\begin{aligned}
& P_{p v_{t}}=P_{p v, p_{t}}-S_{p v_{t}} \\
& P_{p v_{t}}^{\text {pred }}-\sigma_{p v}^{\text {down }} \leq P_{p v, p_{t}} \leq P_{p v_{t}}^{p r e d}-\sigma_{p v}^{u p} \\
& 0 \leq S_{p v_{t}} \leq P_{p v, p_{t}}
\end{aligned}
$$

Here, $P_{p v_{t}}$ is the power output of the PV system. Equation (5) represents the maximum and minimum power limitations of PV system. $S_{p v_{t}}$ is the spillage power of the PV system. The potential power generation for the PV system is limited to maximum and minimum bands due to the prediction of the PV power generation as represented in (5). Here, $\sigma_{p v}^{d o w n}$ and $\sigma_{p v}^{u p}$ are down and up prediction variances for PV system, respectively. Also, $P_{p v_{t}}^{p r e d}$ is the predicted power generation for PV system. The spillage power is the amount of PV power generation that is spilled. In other words, the PV system can potentially generate it but DEMS cannot operate it because of the economic and technical constraints. This amount is positive or equal to zero, and is limited to the actual power generation of PV, $P_{p v, p_{t}}$, as represented in (6). 


\section{B. Electrical Loads}

Electrical loads include loads that can be controllable and/or shiftable. In this paper, three types of loads are modelled. Space heater, $L_{s h_{t}}$, which is a controllable load, storage water heater, $L_{s w h_{t}}$, which is a shiftable load, and must-run services, $L_{m r s_{t}}$, which are non-controllable-shiftable loads. Equations (7) and (8) define total electrical load and total load shedding, respectively. These loads are described in the following subsections.

$$
\begin{aligned}
& \sum_{j \in E L s} L_{j_{t}}=L_{s h_{t}}+L_{s w h_{t}}+L_{m r s_{t}} \\
& \sum_{j \in E L s} L_{j_{t}}^{\text {shed }}=L_{s h_{t}}^{\text {shed }}+L_{\text {swh }}^{\text {shed }}+L_{m r s_{t}}^{\text {shed }}
\end{aligned}
$$

1) Space Heater: The space heater provides the desired indoor temperature. There is a differential equation between the indoor temperature and the electrical demand of the space heater device. Equation (9) represents the performance of the space heater based on the relationship of the indoor temperature with its electrical load. In (9), $\theta_{0}$ is the initial indoor temperature which is assumed to be equal to the desired temperature. Equation (10) represents the indoor temperature that is limited to $1^{\circ} \mathrm{C}$ more or less than the desired temperature. Also, the maximum and minimum constraints of the space heater load are stated in (11). Besides, the load shedding limitation of the space heater is represented in (12).

$$
\begin{aligned}
& \theta_{\text {in }}+1 \\
& +\theta_{i n_{t}} e^{-1 / R C}+L_{s h_{t}} R\left(1-e^{-1 / R C}\right) \\
& +\theta_{\text {out }_{t}}^{\text {pred }}\left(1-e^{-1 / R C}\right), t \geq 2 \\
& \theta_{\text {in }}=\theta_{0}=\theta_{\text {des }}, t=1 \\
& -1 \leq \theta_{\text {in }}-\theta_{\text {des }} \leq 1 \\
& L_{\text {sht }_{t}}^{\text {min }} \leq L_{s h_{t}} \leq L_{\text {sh }}^{\text {max }} \\
& 0 \leq L_{\text {shed }}^{\text {shed }} \leq L_{s h_{t}}
\end{aligned}
$$

2) Storage Water Heater: Storage water heater is in charge of storing the heat in the water tank. The maximum and minimum limitations of the storage water heater's load and energy consumption are stated in (13) and (14), respectively. The load shedding constraint related to the storage water heater is represented in (15).

$$
\begin{aligned}
& L_{s w h_{t}}^{\min } \leq L_{s w h_{t}} \leq L_{s w h_{t}}^{\max } \\
& \sum_{t=1}^{N_{t}} L_{s w h_{t}}=U_{s w h_{t}} \\
& 0 \leq L_{\text {shed }_{t}}^{\text {shed }} \leq L_{\text {swh }}
\end{aligned}
$$

3) Must-run Services: Must-run services consist of loads that should be provided quickly - e.g. lighting, entertainment, etc. In this paper, it is assumed that there is no uncertainty in predicting the electrical loads of must-run services. Also, the load shedding constraint is stated in (17).

$$
\begin{aligned}
& L_{m r s_{t}}=L_{m r s_{t}}^{\text {pred }} \\
& 0 \leq L_{m r s_{t}}^{\text {shed }} \leq L_{m r s_{t}}
\end{aligned}
$$

\section{Battery System}

The battery system can be used to apply the charge and discharge strategies in the DEMS. The proposed strategy for the operation of the battery in the DEMS, follows the algorithm shown in Fig. 2. Based on this strategy, the main purpose of the system is to provide the domestic electrical demand locally. In this case, the surplus of the PV power generation is stored in the battery. Then, the DEMS will sell the power to the grid if the battery is charged completely. On the other hand, the battery system discharges if the electrical demand is more than the power generation of the PV. Besides, if the battery cannot provide all the required electrical load, the DEMS will buy the electricity from the power grid as depicted in Fig. 2.

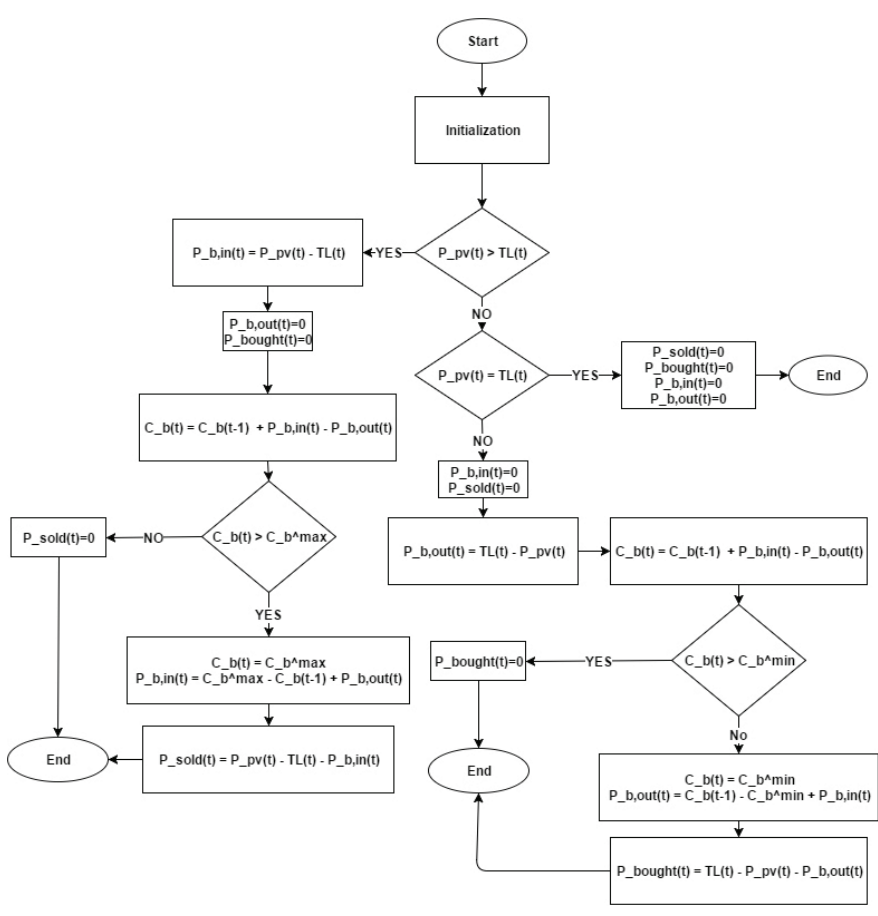

Fig. 2: Flowchart modelling the Battery parameters

\section{ARTIFICIAL ImMUNE Systems}

The immune system (IS) is present in the organisms of many species, protecting them from harmful external agents. In the case of vertebrates, the immune system is composed of diverse molecules, cells and organs distributed in the body, however, they are not controlled by any central entity. All the elements found in the immune system are called antigens. When the antigen originates from within the internal organism, it protects the body and is called a self-antigen (or simply self). Antigens from external environments can provoke different diseases and are denominated as non-self -antigens. The immune system is responsible for distinguishing between self-antigens and nonself-antigens, by a pattern recognition process and attacking the harmful non-self antigens [15].

Inspired by the natural immune system behavior, [16] presents the CLONALG algorithm, a clonal selection proce- 
dure that performs pattern recognition. This algorithm allows to mutate some antibodies according to their affinity to an external antigen. To do so, it generates copies of the antibodies according to their affinity with the antigen. The copies are mutated with a rate $\delta$ inversely proportional to their affinity with the antigen, based on the Equation (18).

$$
\delta=\frac{e^{f_{i}}}{\beta}
$$

where $\beta$ is a constant obtained empirically to normalize the effect of the fitness rate $f_{i}$ obtained by each cell. These new individuals are added to the general population and reevaluated to be reproduced and mutated again. Thus, based upon an evolutionary-like behavior, CLONALG learns how to recognize patterns [16].

In order to adapt the immune behavior to optimization problems, a new formulation is developed by [14], called opt-aiNet. The information provided is represented through the antigens to be recognized by the antibodies. We define fitness as the the affinity between the antigen and the antibody. Hence, high fitness values reflect high affinity. Also, fitness can be compared with a distance metric between antigen and antibody. Small distances represent high affinity, while long distances mean low affinity.

In the first stage, the antibodies are randomly generated. The antibodies are presented to the antigens to calculate the affinity between them. The affinity is measured with a distance metric such as the Euclidean distance, so opt-aiNet is capable of optimizing functions in $\mathbb{R}^{N}$. Those with high affinity are selected and reproduced based on their fitness value according to the CLONALG algorithm. In order to preserve diversity, antibodies whose affinity is lower than a given threshold $t_{s}$ are removed from the population. The corresponding pseudocode is presented in Alg. (1).

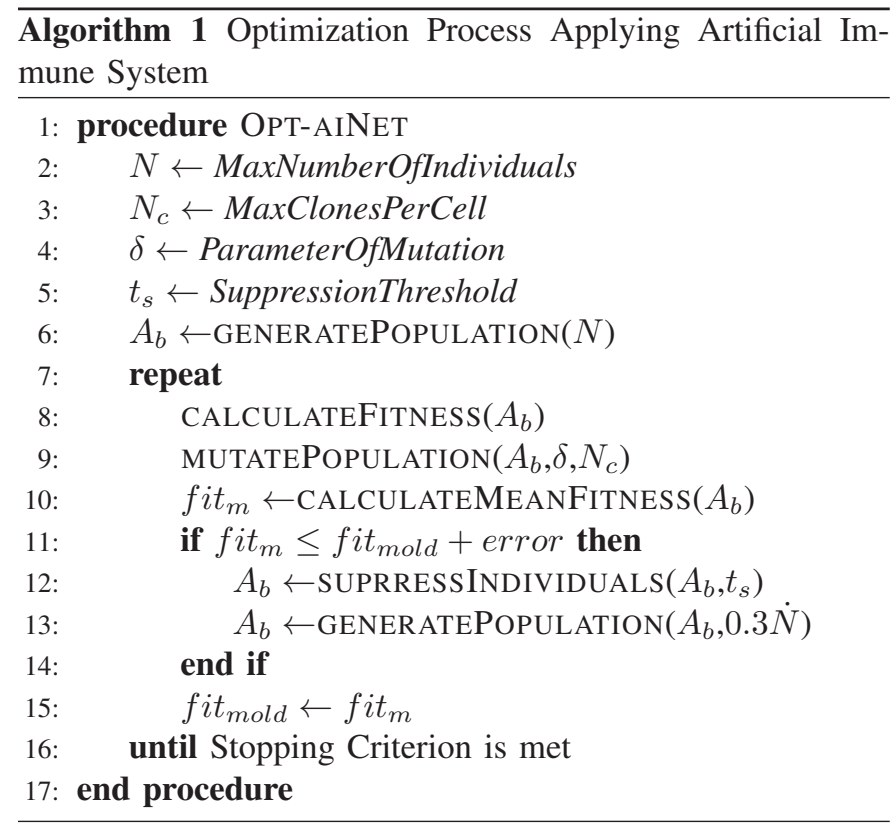

The most important features of opt-aiNet are:
- Its ability to find several optima of the objective function in parallel, meanwhile preserving diversity of the solutions. This means that opt-aiNet can find a set of good candidates for the solution of the optimization problems which are different from one another.

- Its ability to memorize to preserve those individuals that are good enough to be reproduced and mutated in consecutive iterations

Opt-ainet was applied in different contexts with positive results [17], [18], [19]. In former applications, opt-aiNet worked with a low number of variables (each individual contains about 6 variables as much) and without constraints encoded as mathematical functions. In the present work, we adjust this algorithm to be applied to an electrical problem. To this end, opt-Ainet was modified to admit up to 336 variables and 25 linear constraints (inequalities and equations).

\section{EXPERIMENTAL SETTING}

To assess the performance of the proposed DEMS, the physical system from [7] is applied. However, some modifications of the system parameters are made. The maximum power produced by the PV system is $2-\mathrm{kW}$. The battery can store between 0.48 and $2.4 \mathrm{kWh}$. Maximum heating power of the Space Heater (SH) equals $2 \mathrm{~kW}$ to maintain the temperature of the house within \pm 1 of desired temperature $\left(23^{\circ} \mathrm{C}\right)$. The thermal resistance, $\mathrm{R}$, of the building shell is equal to $18^{\circ} \mathrm{C} / \mathrm{kW}$, and $\mathrm{C}$ equals $0.525 \mathrm{kWh} /{ }^{\circ} \mathrm{C}$. The energy capacity of the Storage Water Heater (SWH) is $10.46 \mathrm{kWh}$ $(180 \mathrm{~L})$ which has $2 \mathrm{~kW}$ heating element. Table I displays the predicted data that has been used in [20]. Table II gives the price data of the system. Moreover, VOLL, and spillage costs of PV power generation are shown in Table III.

All this information is integrated into the main system and considered by the AIS to maximize the function given in Equation (1). This objective function allows the AIS to evaluate, mutate or suppress the individuals. Following the optaiNet procedure, the initial population is randomly generated, always accomplishing the constraints modelled in the electrical management problem. The steps to follow by our specific AIS are detailed below:

1) Initiate $N$ population following the linear constraints and the flowchart if it is the case.

2) Evaluate each individual according to the optimization function given in Equation 1.

3) Create $N_{c}$ clones of each individual. The elements of each clone should be slightly changed according to the mutation equation (Equation 18). This mutation procedure should guarantee that all the clones accomplish the linear constraints and the flowchart.

4) For each cell or antibody, select the best clone with the highest fitness value.

5) If the mean fitness of the last iteration and the present one are below a limit, then we suppress similar individuals, according to the similarity threshold $t_{s}$ that measures distances between two antibodies. 
TABLE I: Predicted Data of Uncertain Variables

\begin{tabular}{llllll}
\hline $\mathrm{t}$ & $P_{p_{t}}^{\text {pred }}$ & $\sigma_{p v}^{\text {up }}$ & $\sigma_{p v}^{\text {down }}$ & $\theta_{\text {out }_{t}}^{\text {red }}$ & $L_{\text {mrst }}^{\text {pred }}$ \\
\hline 1 & 0 & 0.03 & 0.01 & 5.5 & 0.3 \\
2 & 0 & 0.03 & 0.01 & 5.5 & 0.3 \\
3 & 0 & 0.03 & 0.01 & 5.2 & 0.3 \\
4 & 0 & 0.03 & 0.01 & 5.2 & 0.3 \\
5 & 0 & 0.03 & 0.01 & 4.8 & 0.4 \\
6 & 0 & 0.03 & 0.01 & 5.5 & 0.6 \\
7 & 0.25 & 0.03 & 0.01 & 6.5 & 0.8 \\
8 & 0.75 & 0.03 & 0.01 & 7.5 & 0.8 \\
9 & 1.25 & 0.03 & 0.01 & 9.8 & 0.7 \\
10 & 1.75 & 0.03 & 0.01 & 10.1 & 0.55 \\
11 & 1.9 & 0.03 & 0.01 & 11.5 & 0.5 \\
12 & 1.9 & 0.03 & 0.01 & 12 & 0.5 \\
13 & 1.9 & 0.03 & 0.01 & 12.5 & 0.5 \\
14 & 1.75 & 0.03 & 0.01 & 12 & 0.5 \\
15 & 1.25 & 0.03 & 0.01 & 11.5 & 0.6 \\
16 & 0.75 & 0.03 & 0.01 & 10 & 0.8 \\
17 & 0.25 & 0.03 & 0.01 & 9 & 1.5 \\
18 & 0 & 0.03 & 0.01 & 8.5 & 1.8 \\
19 & 0 & 0.03 & 0.01 & 8 & 1.7 \\
20 & 0 & 0.03 & 0.01 & 7.5 & 1.1 \\
21 & 0 & 0.03 & 0.01 & 7 & 0.9 \\
22 & 0 & 0.03 & 0.01 & 6.5 & 0.7 \\
23 & 0 & 0.03 & 0.01 & 6.2 & 0.6 \\
24 & 0 & 0.03 & 0.01 & 6 & 0.4 \\
\hline & & & & & \\
\hline
\end{tabular}

TABLE II: PRICE DATA OF THE SYSTEM

\begin{tabular}{|l|c|c|}
\cline { 2 - 3 } \multicolumn{1}{c|}{} & \multicolumn{2}{c|}{ Price $(\$ / \mathrm{MW})$} \\
\hline $\begin{array}{c}\text { Time } \\
\text { (hour) }\end{array}$ & $\lambda_{i}$ & $\lambda_{\text {net }}$ \\
\hline $23-7$ & 2.2 & 0.0814 \\
\hline $8-14$ & 2.2 & 0.1408 \\
\hline $15-20$ & 2.2 & 0.3564 \\
\hline $21-22$ & 2.2 & 0.1408 \\
\hline
\end{tabular}

TABLE III: VOLL AND SPILLAGE COSTS

\begin{tabular}{|c|c|c|c|c|}
\cline { 2 - 5 } \multicolumn{1}{c|}{} & \multicolumn{3}{c|}{ VOLL (\$/MW) } & Spillage Cost (\$/MW) \\
\hline $\begin{array}{c}\text { Time } \\
\text { (hour) }\end{array}$ & SH & SWH & MRS & PV \\
\hline $22-7$ & 1 & 1 & 2.2 & 4 \\
\hline $8-21$ & 1 & 1 & 2.2 & 4 \\
\hline
\end{tabular}

6) If we suppressed some individuals, then we have to add new random population. These new solutions are generated following the constraints and the flowchart if that is the case.

7) This work flow is repeated until convergence criterion (maximum number of iterations gen). The result is one or more individuals with an optimum objective value.

AIS needs some parameters to be set beforehand in order to optimize a problem correctly. These parameters are related to the clonation and mutation process, the suppression algorithm and the convergence criterion. For each iteration, a number of clones $N_{c}$ are generated per each cell. This number $N_{c}$ is set empirically, and can influence the final results. Generally, if we set $N_{c}$ with a very low value, we can delay the convergence criterion, as we are not able to find enough diversity to select better individuals for each cell. Otherwise, if we generate too many clones, the time upon convergence might be longer than expected.

The mutation process (Equation (18)) depends on the mutation constant $\beta$, which measures the influence of the fitness value on the mutation of different clones. If $\beta$ is set to a very high value, the individuals can be randomly mutated, as the fitness values are not influenced by the mutation process. Otherwise, if $\beta$ is very low the individuals are very strongly mutated, which can make our final results biased.

The suppression constant $t_{s}$ is related to the minimum value for similarity between two individuals. If it is set to a very low value, the list of similar individuals can be largely reduced and the population can augment exponentially, which influences the time upon convergence. Otherwise, if $t_{s}$ is very high, the population can decrease exponentially and render a false convergence upon a false optimum value.

Finally, the convergence criterion depends on the number of maximum iterations gen and the population $N$. If we set a few iterations or if the initial population $N$ is very low, the algorithm might not converge correctly and give false optima values. Otherwise, the time cost can be very high and not desirable for our problem.

As we stated in the Introduction, two different strategies have been followed. On the one hand, we optimize the profit when the battery is not considered (Strategy 1). On the other hand, we consider the whole system with the battery, following the flowchart of Figure 2 (Strategy 2). In this strategy, two different situations can happen: the battery is available, meaning that it can be filled and used when necessary, or unavailable, when the battery is full and cannot be charged or discharged under any circumstances. Given both scenarios, we measure how the most important parameters of the AIS can influence the optimization process considering the time elapsed to find the optima and the maximum fitness value, and adjust them to obtain the best results. In order to obtain an efficient system, we need to find a balance between time and fitness.

To this end, we gradually change the different parameters between an interval. $N$ oscillates between 10 and 350, and $N_{c}$ between 4 and 20. The maximum number of generation is set between 10 and 500 . The suppression and mutation parameters depend on the fitness values, so they would be adjusted to the range of fitness values retrieved. Therefore, in Strategy $1 t_{s}$ changes from 5 to 20 and $\beta$ takes values from 10 to 100 , while in Strategy $2, t_{s}$ goes from 1 to 15 and $\beta$ oscillates between 0.5 and 5.

We finally set the AIS parameters according to Table IV, which gave the optima performance in terms of fitness and time following the discussion above.

Once we have described the AIS configuration with all the data integrated, the results of the different simulations will be provided in Section V. 


\begin{tabular}{c|ccccc} 
& $N$ & $N_{c}$ & gen & $t_{s}$ & $\beta$ \\
\hline Strategy I & 250 & 12 & 250 & 10 & 100 \\
Strategy II & 250 & 18 & 300 & 3 & 10
\end{tabular}

TABLE IV: Optima values set for $N, N_{c}$, gen, $t_{s}$ and $\beta$ in both strategies.

\section{Simulation Results}

The evaluation is twofold. On the one hand, we aim to demonstrate that AIS obtains positive results for optimization problems in smart grids. To this end, a comparative study between a classical genetic algorithm and AIS is performed. On the other hand, we aim to analyze the impact of the energy management strategy when the flowchart in Figure 2 is accomplished. Finally, we want to demonstrate the impact that the battery can have in getting a maximum profit from a the domestic environment. Thus, we distinguish three different case studies:

- Case Study I: Comparative Study between GA and AIS in Strategy 1.

- Case Study II: Comparison between Strategy 1 and Strategy 2.

- Case Study III: Analysis of Strategy 2 when the battery is disconnected or connected.

In Case Study I, we aim to compare the results between GA and AIS. In this case, the model considered does not involve parameters corresponding to the battery charge, neglecting the flowchart shown in Figure 2.

In Case Study II, we optimize the parameters related to the PV system, the space heater, the water heater and the mustrun services. Just as in Case Study I, the parameters related to the battery charge are not considered. We aim to optimize all the parameters stated in the previous section for 24 hours, thus, each individual of the AIS will consist of a vector of 264 elements with equality and inequality constraints. In order to analyze the impact of the energy management strategy, this optimization method is then compared with the Strategy 2 when the battery is disconnected.

Finally, in Case Study III, individuals in the AIS have 336 elements because the parameters corresponding to the battery charge and load are set as elements of optimization. Each individual will be constructed following the linear constraints and the flowchart before being plunged into the population. We performed two different analysis: when the battery was disconnected, meaning that the parameters related to the battery charge and the battery load are set to 0 in all cases (although the flowchart is followed in any case), and when the battery was connected, meaning that all the variables are considered and their value can change.

\section{A. Comparison between GA and AIS}

In order to demonstrate the efficiency of the Artificial Immune System in the energy management optimization problem, we performed a comparative test with a classical genetic algorithm. The goal is to predict the optimum values for each variable during 24 hours, following Strategy 1. We applied
TABLE V: Results of the Objective Function for three different configurations of the GA.

\begin{tabular}{|c|c|c|c|}
\hline Parameters & $\mathrm{GA}_{1}$ & $\mathrm{GA}_{2}$ & $\mathrm{GA}_{3}$ \\
\hline Mutation Rate & 0.2 & 0.3 & 0.35 \\
\hline Cross-over Rate & 0.7 & 0.8 & 0.75 \\
\hline Selection Function & Roulette & Tournament & Tournament \\
\hline Number of generations & 2000 & 2800 & 3000 \\
\hline Objective Value & 22.92 & $\mathbf{2 3 . 5 5}$ & 23.06 \\
\hline AIS Obj. Value & \multicolumn{3}{|c|}{$\mathbf{2 3 . 8 6}$} \\
\hline
\end{tabular}

the linear constraints proposed in the electrical model, and we measured the objective function for the optimized variables.

The GA contains some parameters that can be set for an optimal operation. In this preliminary study, we set the mutation and cross-over rates, the selection function and the number of generations. Table V shows three different performances with three different configurations of the GA.

As we can see from the objective value obtained, $\mathrm{GA}_{2}$ gets the best results. However, in any of the configurations, the results improve when AIS with its best configuration is applied. However, more parameters of the GA could be modified to improve the current performance and make the comparison more realistic. For future work, we believe that a more in-depth analysis of the GA is necessary.

\section{B. Impact of Energy Management Strategy}

In this section, two proposed strategies for domestic energy management problem are evaluated. As putlined before, maximizing the home's energy profit is the main goal of the first strategy. However, the main purpose of the second strategy is to maximize energy profit and act as an autonomous energy system. In this section, the battery system is not considered. As shown in Table VI, the value of the objective function in Strategy 1 is more that of Strategy 2. However, the transacted energy between home and power grid is less in Strategy 2, because we pursue the autonomous management of energy at home.

TABLE VI: Impact of energy management strategies on the amount of sold/ bought electrical energy to/from power grid and $\mathrm{OF}$.

\begin{tabular}{|l|c|c|}
\cline { 2 - 3 } \multicolumn{1}{c|}{} & Strategy 1 & Strategy 2 \\
\hline OF & 23.8613 & 5.11 \\
\hline$E_{\text {sold }}$ & 14.22 & 4.64 \\
\hline$E_{\text {bought }}$ & 45.66 & 26.53 \\
\hline
\end{tabular}

\section{Impact of Battery}

The impacts of a battery system on the objective function and exchanged energy are assessed based on strategy 2. From the data in Table VII, we can see that the battery system can improve the value of the objective function. Table VII also considered a situation in which the battery increases the amount of electrical energy sold from the smart home to the grid, and it decreases the amount of home's electrical energy bought from the network. 
TABLE VII: impact of battery system on the amount of sold/ bought electrical energy to/from power grid and OF.

\begin{tabular}{|l|c|c|}
\cline { 2 - 3 } \multicolumn{1}{c|}{} & \multicolumn{2}{c|}{ Strategy 2} \\
\cline { 2 - 3 } \multicolumn{1}{c|}{} & with battery & without battery \\
\hline$O F$ & 12.31 & 5.11 \\
\hline$E_{\text {sold }}$ & 6.22 & 4.64 \\
\hline$E_{\text {bought }}$ & 10.47 & 26.53 \\
\hline
\end{tabular}

\section{CONCLUSION}

Residential buildings in smart grids have an important role in the optimization of energy scheduling. In order to optimize such problems, here we applied an Artificial Immune System based on Opt-aiNet, an optimization version of an AIS used in different contexts [14]. In this work we show how an AIS can be used to solve a power system optimization problem efficiently. To this end, we have adapted the opt-aiNet algorithm to include complex constraints in the optimization problem and to work with a large number of variables efficiently.

From an electrical point of view, we analyze two different strategies. While maximizing the energy profit of a home is the main goal of the first strategy, the main purpose of the second strategy is to maximize its energy profit and act as an autonomous energy system simultaneously and independently of whether the battery variables are considered or not. In both cases, the parameters applied to the AIS to manage the mutation, clonation or convergence criteria are fully analyzed in order to optimize the performance of the algorithm. Once the best parameters are set, the final results show that the application of the battery increases the efficiency of the model. We performed three different case studies. Firstly, we demonstrated of the AIS by comparing it with three different configurations of a genetic algorithm. Secondly, we compare both strategies to highlight the advantages of including a battery in our system. Finally, we analyze the impact of the battery considering two different situations: when the battery is available (can be filled and used in our system) or unavailable (the battery is full and cannot be used). This last comparison shows the importance of using a battery for improving the general profit of our residential electrical system.

Future work will consist of improving the results of the optimization problem with the GA and presenting a more complex case with non-linear constraints as well as considering the uncertainty of predicted variables to encourage the use of evolutionary computing.

\section{ACKNOWLEDGMENTS}

This work has been partially supported by the Spanish Government through the European Commission H2020 MSCARISE-2014: DREAM-GO Project (Marie Sklodowska-Curie grant agreement No 641794)

\section{REFERENCES}

[1] M. A. A. Pedrasa, T. D. Spooner, and I. F. MacGill, "The value of accurate forecasts and a probabilistic method for robust scheduling of residential distributed energy resources," in Probabilistic Methods
Applied to Power Systems (PMAPS), 2010 IEEE 11th International Conference on. IEEE, 2010, pp. 587-592.

[2] A. Molderink, V. Bakker, M. G. Bosman, J. L. Hurink, and G. J. Smit, "Domestic energy management methodology for optimizing efficiency in smart grids," in PowerTech, 2009 IEEE Bucharest. IEEE, 2009, pp. $1-7$.

[3] M. Nistor and C. Antunes, "Integrated management of energy resources in residential buildings-a markovian approach," IEEE Transactions on Smart Grid, 2016.

[4] S. Althaher, P. Mancarella, and J. Mutale, "Automated demand response from home energy management system under dynamic pricing and power and comfort constraints," IEEE Transactions on Smart Grid, vol. 6, no. 4, pp. 1874-1883, 2015.

[5] T. Hansen, E. Chong, S. Suryanarayanan, A. Maciejewski, and H. Siegel, "A partially observable markov decision process approach to residential home energy management," IEEE Transactions on Smart Grid, 2016.

[6] J. L. Fernandez-Marquez, G. D. M. Serugendo, S. Montagna, M. Viroli, and J. L. Arcos, "Description and composition of bio-inspired design patterns: a complete overview," Natural Computing, vol. 12, no. 1, pp. 43-67, 2013.

[7] M. A. Pedrasa, E. Spooner, and I. MacGill, "Improved energy services provision through the intelligent control of distributed energy resources," in PowerTech, 2009 IEEE Bucharest. IEEE, 2009, pp. 1-8.

[8] A. Soares, C. H. Antunes, C. Oliveira, and Á. Gomes, "A multi-objective genetic approach to domestic load scheduling in an energy management system," Energy, vol. 77, pp. 144-152, 2014.

[9] B. Yuce, Y. Rezgui, and M. Mourshed, "Ann-ga smart appliance scheduling for optimised energy management in the domestic sector," Energy and Buildings, vol. 111, pp. 311-325, 2016.

[10] L. dos Santos Coelho and V. C. Mariani, "Chaotic artificial immune approach applied to economic dispatch of electric energy using thermal units," Chaos, Solitons \& Fractals, vol. 40, no. 5, pp. 2376-2383, 2009.

[11] F. Alonso, D. Oliveira, and A. Z. de Souza, "Artificial immune systems optimization approach for multiobjective distribution system reconfiguration," IEEE Transactions on Power Systems, vol. 30, no. 2, pp. 840$847,2015$.

[12] J. Zhu, F. Lauri, A. Koukam, V. Hilaire, and M. G. Simoes, "Improving thermal comfort in residential buildings using artificial immune system," in Ubiquitous Intelligence and Computing, 2013 IEEE 10th International Conference on and 10th International Conference on Autonomic and Trusted Computing (UIC/ATC). IEEE, 2013, pp. 194-200.

[13] K. Lakshmi and S. Vasantharathna, "Gencos wind-thermal scheduling problem using artificial immune system algorithm," International Journal of Electrical Power \& Energy Systems, vol. 54, pp. 112-122, 2014

[14] L. N. De Castro and J. Timmis, "An artificial immune network for multimodal function optimization," in Evolutionary Computation, 2002. CEC'02. Proceedings of the 2002 Congress on, vol. 1. IEEE, 2002, pp. 699-704.

[15] _ - Artificial immune systems: a new computational intelligence approach. Springer Science \& Business Media, 2002.

[16] L. N. De Castro and F. J. Von Zuben, "The clonal selection algorithm with engineering applications," in Proceedings of GECCO, vol. 2000, 2000, pp. 36-39.

[17] L.-F. Hsu, C.-C. Hsu, and T.-D. Lin, "An intelligent artificial system: artificial immune based hybrid genetic algorithm for the vehicle routing problem," Applied Mathematics \& Information Sciences, vol. 8, no. 3, p. 1191, 2014.

[18] S. Shamshirband, N. B. Anuar, M. L. M. Kiah, V. A. Rohani, D. Petković, S. Misra, and A. N. Khan, "Co-fais: cooperative fuzzy artificial immune system for detecting intrusion in wireless sensor networks," Journal of Network and Computer Applications, vol. 42, pp. 102-117, 2014.

[19] M. Navarro, M. Caetano, G. Bernardes, L. N. de Castro, and J. M. Corchado, "Automatic generation of chord progressions with an artificial immune system," in International Conference on Evolutionary and Biologically Inspired Music and Art. Springer, 2015, pp. 175-186.

[20] F. P.-C. A. Shokri Gazafroudi and J. M. Corchado, "Residential energy management using a novel interval optimization method," in 4th International Conference on Control, Decision and Inf. Tech. (CoDIT'17). IEEE, 2017. 